\title{
Using Topical Applications of TamoxifenandaCombination of Phytonutrients Based on Breast MRI to Inhibit Estrogen-Related Proliferation of Human Breast Tissue
}

\author{
Shoei-Loong Lin ${ }^{1}$, Cheuk-Sing Choy ${ }^{2}$, Wing P Chan ${ }^{3}$ and Ting-Kai Leung ${ }^{3,4 *}$ \\ ${ }^{1}$ Department of Surgery, Taipei Hospital, Ministry of health and Welfare, Taiwan., R.O.C and Department of Surgery, School of Medicine, College of Medicine, Taipei Medical \\ University, Taiwan \\ ${ }^{2}$ Emergency and intensive care department, Taipei Hospital, Department of Health, Taiwan \\ ${ }^{3}$ Department of Radiology, School of Medicine, College of Medicine, Taipei Medical University and Department of Radiology, Wan Fang Hospital, Taipei Medical University, \\ Taiwan \\ ${ }^{4}$ Diagnostic Radiology department, Taipei Hospital, Ministry of Health and Welfare, Taiwan
}

\begin{abstract}
Objective: In January of 2013, the National Institute of Health and Clinical Excellence of United Kingdom have announced that taking tamoxifen for five years could reduce breast cancer risk, include positive family history or chronic estrogen and phytoestrogen users. However, routinely oral intake of tamoxifen for breast cancer prophylaxis on high risk women is occasionally difficult because of its potential side effects. The intention of this study is to prove transdermal delivery by using minimal dose of tamoxifen and phytonutrients for breast protection.
\end{abstract}

Methods: We retrospectively studied 2795 breast MRI images from Taiwanese women and classified them as either healthy or unhealthy according to BI-RADS categories. A subgroup of the study patients had received estrogen supplements containing estrogen components or phytoestrogen for at least 3 months. Based on breast MRI analysis, these 2 groups were compared and found significant different. The overuse of estrogen or phytoestrogen supplements can increase breast glandular tissue proliferation, as reflected in MRI images. Such proliferation may increase the risk of future breast cancer. We also studied the efficacy of tamoxifen and the phytonutrients to inhibit proliferation of mammary glandular cell, as well as their transdermal absorption in animal models.

Results: We demonstrated cell proliferative change of normal breast cells (MCF-10a) under estrogen treatment For studied of transdermal absorption by topical application, we used Franz cell with artificial skin membrane to demonstrate positive transdermal diffusion using animal urine/feces, blood samples and subcutaneous skin retention for HPLC analysis.

Conclusion: We successful demonstrate the transdermal absorption of topical application of tamoxifen and phytonutrients; it may help to reduce breast cancer development in regular estrogen and phytoestrogen users.

Keywords: Breast MRI; Estrogen; Phytoestrogen; Proliferation; Franz cell; Tamoxifen; Phytonurtrients; Transdermal absorption

\section{Introduction}

Numerous studies have demonstrated that hormonal replacement therapy (HRT; estrogen plus progesterone) increases the risk of breast cancer [1-5]. Since 2000 the use of HRT has decreased substantially in the general population of some Western countries, such as Germany. It was found a corresponding decline, noted in breast cancer incidence between 2000 and 2005 [6]. This finding clearly reflects the positive relationship between HRT and the incidence of breast cancer [7]. Observations from the Nurses' Health Study (NHS) [8] and the Million Women Study (MWS) [2] suggest an association between estrogen and breast cancer [9]. By contrast, the Women's Health Initiative (WHI) [1] did not detect a higher level of risk in an estrogen-only (estrogen replacement therapy; ERT) group. Beral et al. [10] suggested that HRT is associated with a greater increase in the risk of breast cancer than ERT [10], suggesting the possibility of additional mechanisms involved. When considering the risk of breast cancer and the associated benefits of ERT in postmenopausal women, the impact of ERT on the quality of life, bone metabolism, and cardiovascular functionality should be assessed because the benefits associated with ERT may outweigh the risks in healthy postmenopausal women [11]. Determining such risks and associated benefits is difficult, and depends on individual circumstances [12]. The mechanisms of initiating versus promoting hormone-sensitive cancer types, particularly of breast cancer, are not fully understood [13-15].

Many Taiwanese women have been restrained from using ERT because they were alerted of the risk of breast cancer. However, it is a common misconception that plant-based food supplements (such as phytoestrogen) and Chinese herbs assist postmenopausal women in avoiding breast cancer risks, especially among Taiwanese women [16-18]. Furthermore, refraining from estrogen, plant-based foods, and Chinese herb use does not guarantee that women will be free from exogenous estrogen influences. Environmental estrogen pollution is also a factor because steroidal estrogen is constantly excreted into the

${ }^{*}$ Corresponding author: Ting Kai Leung, Department of Radiology, Taipe Hospital, Ministry of Health and Welfare, NO.127, Su Yuan Road, Hsin Chuang District, New Taipei City, 242-13, Taiwan, Tel: 886-2-22765566+2187; Fax: 8862-22765591; E-mail: hk8648@tmu.edu.tw

Received December 03, 2013; Accepted January 07, 2014; Published January 13, 2014

Citation: Lin SL, Choy CS, Chan WP, Leung TK (2014) Using Topical Applications of Tamoxifen and a Combination of Phytonutrients Based on Breast MRI to Inhibit Estrogen-Related Proliferation of Human Breast Tissue. Pharm Anal Acta 5: 281. doi: 10.4172/2153-2435.1000281

Copyright: (c) 2014 Lin SL, et al. This is an open-access article distributed under the terms of the Creative Commons Attribution License, which permits unrestricted use, distribution, and reproduction in any medium, provided the original author and source are credited. 
environment. Pregnant women may excrete $10 \mu \mathrm{mol} / \mathrm{d}$ of estrogen, including estradiol and estrone. Chicken manure can contain more than $1 \mu \mathrm{mol} / \mathrm{g}$ of hormones (including estrogen), and other animals, such as cows, swine, horses, and goats, also excrete large amounts of estrogen. It is possible that environmental estrogen observed in water sources such as lakes and other water bodies can contaminate public drinking water supplies [19].

According to our clinical observation, magnetic resonance imaging (MRI) of the breast is extremely sensitive in detecting lesions and can identify the abnormal statuses of proliferation, angiogenesis, and microvascular permeability that occur in early breast neoplasm formation. In this study, we retrospectively reviewed breast MRI images of patients with a known estrogen and phytoestrogen intake to investigate a possible associated cancer risk. We used MRI results to assess the effect of estrogen and phytoestrogen on breast glandular tissue.

Applying tamoxifen on normal women with a risk of breast cancer has already been advocated [20,21]. In January of 2013, The National Institute of Health and Clinical Excellence of United Kingdom have further announced that taking tamoxifen for five years could reduce high risk group with family history of the disease and chronic estrogen users. However, using tamoxifen to inhibit endogenous or exogenous estrogen effects is occasionally difficult because of its potential side effects. Since its introduction for clinical use in the early 1970s, synthetic anti-estrogen tamoxifen citrate has been shown to contribute to controlling human breast cancer and recurrence. Although its mechanisms of action and pharmacology are not completely understood, tamoxifen appears to act predominantly by blocking the action of estrogen by binding to ERs. Clinical trials of tamoxifen for 1 to 2 years in primary breast cancer patients have shown consistent beneficial effects on disease-free survival [22]. However, the gynecologic side effects of tamoxifen are diverse and reflect the complexity of its mechanism of action; the most frequently reported side effects are hot flashes. The most concerning gynecologic side effect is endometrial disease in postmenopausal women [6]. Locally applying tamoxifen to reduce the risk of breast glandular tissue damage caused by estrogen and phytoestrogen is potentially reduces the dosedependence systemic effects of tamoxifen by minimizing its associated adverse effects. Searching for phytonutrients to prevent estrogenicinduced breast cancer is an alternative method for reducing the side effects of using chemical anti-estrogen drugs. According to Veprik et al. [23] combining phytonutrients (carotenoid derivatives, polyphenols, and isothiocyanates) significantly inhibits estrogen response element (ERE) transactivation in breast cancer cells. In this study, we focused on the efficacy of tamoxifen and phytonutrients by examining the in vitro mammary glandular cell-proliferative response to estrogen.

Furthermore, we designed a local drug delivery system for tamoxifen and phytonutrients to develop methods for observing their efficacy of skin penetration and accumulation.

\section{Material and Methods}

\section{Total study population and comparison group}

We collected retrospective data from 2795 MRI images of women who visited the Breast Health Center at Taipei Medical University between December 2008 and July 2012. The retrospective study was approved by an independent ethics committee of our university hospital with certification by the Institutional Review Board (IRB), approval No. 201103011 (Taipei Medical University, Joint IRB). Women who received an MRI around the time of menstruation (3 day before, up to 7 day after) were excluded to reduce the possibility of endogenous estrogen affecting the image results.

\section{Experimental group}

The experimental group formed a subgroup within the total study group. A total of 150 cases of women who had visited the clinic between September 2010 and July 2012 were selected for the experimental group. These women reported having received estrogen supplements containing mimicked estrogen components or estrogen, plant-based foods (such as phytoestrogen) and Chinese herbs, including isoflavone, royal jelly, evening primrose oil, yam extract, four-agent soup (si wu tang), placenta extract, ginseng, and others [24], for at least 3 months prior to their MRI.

\section{Image acquisition}

Pre-enhanced and post-enhanced subtracted images were obtained, and enhanced curve analysis was performed. All imaging studies were performed on a $1.5 \mathrm{~T}$ dedicated spiral breast MRI system (Aurora Systems, USA). For the screening procedure, the patients were placed in a prone position, feet first, on the table inside the DBMRI machine. The breasts were fitted and positioned using a breast transmission-receiving (TR) coil. Off-resonance spiral image mode was used. A gradient echo with $10 \mathrm{~ms}$ of TR and a T2-weighted spin echo with $2.5 \mathrm{~ms}$ of TR were acquired. The echo time was $5 \mathrm{~ms}$ for RODEO and $120 \mathrm{~ms}$ for the T2 sequences. The matrix size was $512 \times 512$, and the slice thickness was 1.1 $\mathrm{mm}$. The field of view of each station was from 20 to $36 \mathrm{~cm}$. Sequences were performed before and after infusing $0.2 \mathrm{mmol} / \mathrm{kg}$ of Magnevist (Bayer-Schering Pharma AG, Germany), administered as a bolus dose with a power injector, followed by a $20 \mathrm{~mL}$ saline flush. Post-processing of data was displayed using Aurora Cad 4.0 (Aurora System, North Andover, MA, USA).

\section{BIRADS grading of breast MRI interpretations}

The Breast Imaging-Reporting and Data System (BI-RADS) was used, and interpreting BRMRI involved adopting the standards of the ACR BIRADS-MRI lexicon [25-27]. Image results scored as either a BIRADS category I or II imply an interpretation of normal and benign findings, with no risk of malignancy. If the image result is scored as category III (probably benign), category IV (suspicious abnormality), category $\mathrm{V}$ (highly suggestive of malignancy), or category VI (malignancy already known), these interpretations indicate that the patient is at risk of malignancy.

\section{Classification of healthy vs. unhealthy breast interpretations}

We used a simple classification system in which the "healthy" images (no risk of malignancy) received an MRI rating of BI-RADS categories I and II, and the "unhealthy" images (with risk of malignancy) were rated as BI-RADS categories III, IV, V, or VI.

\section{Cell culture}

Human mammary epithelial cells (MCF-10A) were purchased from the American Type Culture Collection (BCRC, Taiwan). MCF-10A cells were cultured in phenol red-free low-calcium DMEM/F12 (Gibco, USA) supplemented with Chelex-100-treated (Bio-Rad Laboratories, Richmond, CA) fetal bovine serum (FBS) (10\%). The cell line was plated separately in $75 \mathrm{~cm}^{2}$ culture flasks in a humidified incubator $(5 \%$ $\mathrm{CO}_{2}, 95 \%$ air, $37^{\circ} \mathrm{C}$ ).

The MCF-10A cell line media were changed every 2 days. When the cells approached $85 \%$ to $90 \%$ confluence, they were washed twice with 
Ca- and Mg-free phosphate buffered saline (PBS, $\mathrm{pH} 7.3$ ), and then trypsinized using $0.5 \%$ trypsin and $5.3 \mathrm{mM}$ ethylenediaminetetraacetic acid (EDTA, Gibco-BRL) in PBS for 10 minutes at $37^{\circ} \mathrm{C}$. Trypsinization was stopped by adding a culture medium to the serum. After centrifugation, the dissociated cells were resuspended in the same medium and subcultured in $75 \mathrm{~cm}^{2}$ culture flasks at a ratio of $1: 5$ flasks.

Cell-Proliferative change of MCF-10A under estrogen treatment Observed under a microscope (20X And 100X) and measured using colorimetric XTT

A colorimetric XTT assay is used as an indicator of cell proliferation, which is determined according to its mitochondriadependent reduction to formazan. The cells were plated at a density of $4 \times 10^{6}$ cells/well into 24 -well plates for $24 \mathrm{~h}$, treated with various concentrations of estrogen $(0 \mu \mathrm{M}, 10 \mu \mathrm{M}$, and $100 \mu \mathrm{M})$, and followed by first to third day of treatment. The cells were washed 3 times with PBS, and XTT $(1 \mathrm{mg} / \mathrm{mL})$ was added to the medium for $3 \mathrm{~h}$ before the supernatants were collected. The absorbance was read at $450 \mathrm{~nm}$ by using an spectrophotometer (ELISA, Gemini XPS Molecular Devices, Sunnyvale, CA, USA).

Demonstrating tamoxifen and phytonutrient concentration to inhibit cell proliferation of MCF-10A under the effect of estradiol

Tamoxifen, methanol (HPLC grade), and phosphoric acid (>99\%) were purchased from Sigma-Aldrich (St Louis, MO, USA). The phytonutrients (including epigallocatechin gallate (EGCG), epicatechin gallate (ECG), epigallocatechin (EGC), and epicatechin (EC)) were purchased from the Compson Trading Company, Ltd. (Taipei, Taiwan, ROC).

According to the described method and the result of estradiol concentration, the colorimetric XTT assay was used to demonstrate that tamoxifen concentration is capable of inhibiting the cell proliferation of MCF-10A.

Both tamoxifen $(10 \mu \mathrm{M}$ and $100 \mu \mathrm{M})$ and the phytonutrients (in different concentrations) are used against estradiol concentrations of $100 \mu \mathrm{M}$ and $10 \mu \mathrm{M}$, respectively.

\section{Transdermal and Phytonutrient Absorption Experimental} Model Using Franz Cells with Artificial Skin Membranes

Cellulose acetate membrane discs (Hoefer, USA) were soaked in PBS ( $\mathrm{pH}$ 7.4) for at least $16 \mathrm{~h}$. Cellulose acetate membranes that contained glycerin were rinsed once with a receptor fluid before being placed onto the receptor. The membranes were hydrated and sandwiched between 2 glass microscope slides and submerged in the receptor fluid in a Petri dish to prevent creasing or folding when wet. Air bubbles trapped within the interface of the membrane and receptor were abandoned. The donor compartment was covered and closed tightly by using a pinch clamp.

Using a Franz cell apparatus covered with or without BIOCERAMIC materials, the donor cells were sealed with Parafilm, and the apparatus circulation was maintained at $37^{\circ} \mathrm{C}$ with continuous stirring. An ethanol/phosphate buffer $(50: 50, \mathrm{v} / \mathrm{v})$ was used as a receptor cell buffer for tamoxifen. We collected $200 \mu \mathrm{L}$ of the receptor buffer through the sample port using a pipette, and an equal volume of the buffer was replaced. The drug content was then assessed using HPLC methods, described as follows.

Absolute ethanol (99.9\%) was obtained from Mallinckrodt Baker
B.V. (Deventer, The Netherlands). EMPLURA ${ }^{\circledR}$ (1,2-Propanediol) was purchased from Merck (Huhenbrunn, Germany). We used $10 \mathrm{mg}$ of tamoxifen mixed with $50 \%(\mathrm{v} / \mathrm{v})$ ethanol and $15 \%(\mathrm{v} / \mathrm{v})$ EMPLURA $^{\circledR}$ to form $1 \%$ tamoxifen $(\mathrm{w} / \mathrm{w})$ of the gel for the Franz cell experiment.

\section{HPLC analytical methods}

HPLC analysis was performed using a Waters 2487 HPLC UVVisible Detector (Milford, MA, USA) with a hand injector coupled with 2 Waters 515 pumps, and Waters Millennium 32 software was used for peak data analysis. All of the samples were analyzed using a reversephase C18 column (Inertsil C18 $4.6 \times 250 \mathrm{~mm}, 5 \mu \mathrm{m}$ ). Tamoxifen was detected at $238 \mathrm{~nm}$, the mobile phase ratio $(80: 20)$ of methanol and phosphoric acid $(0.1 \%, \mathrm{v} / \mathrm{v})$ was membrane filtered (Millipore, $0.2 \mu \mathrm{m}$ ) and degassed, and the flow rate was $1 \mathrm{~mL} / \mathrm{min}$. Quantitative measurements obtained using HPLC were expressed in maximum absorbance units (AU) [28]. In UV detectors, a beam of light passes through a certain width of liquid to be analyzed. The detector measures absorbance A of the sample, which is determined as follows:

$A=-\log$ (intensity of light emerging from the sample cell/intensity of light directed on the sample cell).

\section{Analyzing the efficacy of topical use of tamoxifen/ phytonutrients in rat urine}

The tamoxifen cream formulation was composed $10 \%$ by weight of tamoxifen citrate (Nolvadex) (AstraZeneca, London, UK), and the phytonutrients were mixed with $90 \%$ cream base components (paraffin oil/glycerin/DI water), respectively.

Six Wistar rats weighing approximately $300 \mathrm{~g}$ each were obtained from LASCO (Taiwan) and used to examine dermal absorption. Animal care was provided according to an approved protocol and the guidelines of the Taipei Medical University Institutional Animal Care and Use Committee. Conventional-flora rats (male, Wistar rats 150-200 g) were also housed in our own laboratory in metabolic cages $[29,30]$. The Wistar rats were intraperitoneally injected with $30 \mathrm{mg} /$ $\mathrm{kg}$ of Zoletil ${ }^{\mathrm{TM}}$ and $5 \mathrm{mg} / \mathrm{kg}$ Xylazine until anesthesia was complete, and were then placed in the metabolic cages. The metabolic cages were designed to minimize contamination of urine by food and feces and to avoid contamination and dilution of urine by spillage. The dose level of tamoxifen employed in the study was $0.5 \mathrm{mg} / \mathrm{cm}^{2}$, applied as a cream (paraffin oil/glycerin/DI water) and administered to the skin of the shaved back. The treated skin was covered by gauze and protect suit, was used to prevent contamination and rats' intake. The exposure times were 1, 2, 3, 4 and 5 days, and urine and feces were collected on each day. Urine of the collected samples (urine and feces) were selected and were analyzed using HPLC.

\section{Analyzing the efficacy of topical use of tamoxifen/ phytonutrients in rabbit blood}

This dermal absorption study entailed using New Zealand rabbits, which were placed in plastic cages. The dose level of tamoxifen employed in this study was $0.5 \mathrm{~g} / \mathrm{cm}^{2}$, applied as a cream and administered to the skin of the shaved back. The treated skin was covered by gauze to prevent contamination and rabbits' intake. Blood samples from the ears were collected on the first and fourth days. The collected samples were analyzed using HPLC, thus showing successively increased tamoxifen concentration in the blood.

As the analysis on rats' urine above, a similar analysis was also performed on rabbits by using the phytonutrients and one of the main components. 
Citation: Lin SL, Choy CS, Chan WP, Leung TK (2014) Using Topical Applications of Tamoxifen and a Combination of Phytonutrients Based on Breast MRI to Inhibit Estrogen-Related Proliferation of Human Breast Tissue. Pharm Anal Acta 5: 281. doi: 10.4172/2153-2435.1000281

Page 4 of 10

\section{Purification of tamoxifen/phytonutrients from urine and blood, for HPLC Quantitative analysis}

Tamoxifen was added as an internal standard to a $1 \mathrm{~mL}$ urine sample. Twenty milligrams of $2 \mathrm{M} \mathrm{NH}_{4} \mathrm{OAc}(\mathrm{pH} 8.8$ ) were added, and the mixture was allowed to stand overnight at 4TC. After centrifugation, the supernatant was filtered through a Nalgene type $S$ filter unit $(0.2$ gm; Nalgene). The filtrate was slowly applied to a $1.0 \times 10 \mathrm{~cm}$ Amicon PBA-60 boronate affinity column (Amicon), equilibrated with buffer A, then washed with $30 \mathrm{~mL}$ of buffer $\mathrm{A}$, which reduced the absorbance at $254 \mathrm{~nm}$ of the eluate to 0.1-0.3. The column was further washed with $15 \mathrm{~mL}$ of water and then eluted with $25 \mathrm{~mL}$ of $0.1 \mathrm{M}$ HOAc to yield the boronate binding fraction. This fraction, which contained $75 \%-80 \%$ of the radioactive internal standard, was lyophilized and resuspended in $1 \mathrm{~mL}$ of water. Fraction 1, containing thymine glycol and thymidine glycol, was purified using semipreparative HPLC, with a 5-Itm C18 column (Inertsil C18 $4.6 \times 250 \mathrm{~mm}, 5 \mu \mathrm{m}$ ), which was run isocratically in water at $3 \mathrm{~mL} / \mathrm{min}$. Fractions of $2 \mathrm{~mL}$ were collected. Two peaks of urea or another metabolite $(2.7 \mathrm{~min})$ and a tamoxifen metabolite $(4$ min) were pooled separately and concentrated using lyophilization (Savant centrifugal lyophilizer). Each Fraction 1 was further purified using the same column at $2.0 \mathrm{~mL} / \mathrm{min}$ for and $2.5 \mathrm{~mL} / \mathrm{min}$ with water as a mobile phase. Fractions of $0.5 \mathrm{~mL}$ were collected, tamoxifen was determined, and the fractions were pooled to yield tamoxifen Fraction 2 and thymidine glycol Fraction 2. These fractions were lyophilized to dryness in a small test tube.

\section{Determination of tamoxifen/phytonutrients retention after topical application on rat's skin}

The dose level of tamoxifen employed in this study was $10 \mathrm{mg}$, applied as a cream administered to the shaved back of each rat's skin. The treated skins were covered by gauze to prevent contamination and rats' intake. The examination periods were scheduled on the first and fourth days after skin treatment, respectively, and the regional skin was then cleaned to extract possible residual tamoxifen/phytonutrient particles by using distilled water followed by $50 \%$ ethanol. After euthanization and isolating the regional skin, an appropriate, sensitive analytical technique of HPLC (mentioned previously) was then employed to quantify the extracted drug [31,32]. Such a technique was also used to quantify the in vitro retention of tamoxifen/phytonutrient in the full thickness of each rat's skin.

\section{Statistical analysis}

All analyses were performed using SPSS statistical software. We used a one-sample binomial test to compare the outcomes of longterm (at least 3 months) estrogen and phytoestrogen use (experimental group) versus the comparison group (study population). Statistical evaluations of data were performed using a paired $t$ test. Datasets with a $\mathrm{P}$ value of $<0.05$ were considered significant.

\section{Results}

\section{Ratio of healthy to unhealthy images}

We analyzed the results for all 2795 images of women who had received breast MRI screening at our hospital. We classified $50.86 \%$ of the images as healthy (BIRADS I, II); thus, unhealthy images accounted for $48.66 \%$ (BIRADS III, IV, V, VI) of our study population (Table 1 and Figure 1) [7,33-36]. Within the total study group was a subgroup of 219 women who had reported taking estrogen or phytoestrogen regularly for at least 3 months. We retrospectively reviewed their MRI results and classified $34.67 \%$ of the breast images from this subgroup

\begin{tabular}{|l|c|c|c|}
\hline & $\begin{array}{c}\text { Healthy breast } \\
\text { MRI results } \\
\text { (BIRADS I, II) }\end{array}$ & $\begin{array}{c}\text { Unhealthy breast MRI } \\
\text { results } \\
\text { (BIRADS, III, IV, V, VI) }\end{array}$ & $\boldsymbol{P}$ \\
\hline $\begin{array}{l}\text { All women screened } \\
(\mathrm{n}=2795)\end{array}$ & 1360 & 1435 & $0.0010^{\star * *}$ \\
\hline $\begin{array}{l}\text { Estrogen-like group } \\
(\mathrm{n}=150)\end{array}$ & 52 & 98 & \\
\hline
\end{tabular}

*** Statistically significant at $\mathrm{P}<.001$

Table 1: Comparison of MRI images from the study population and a subgroup of estrogen users.
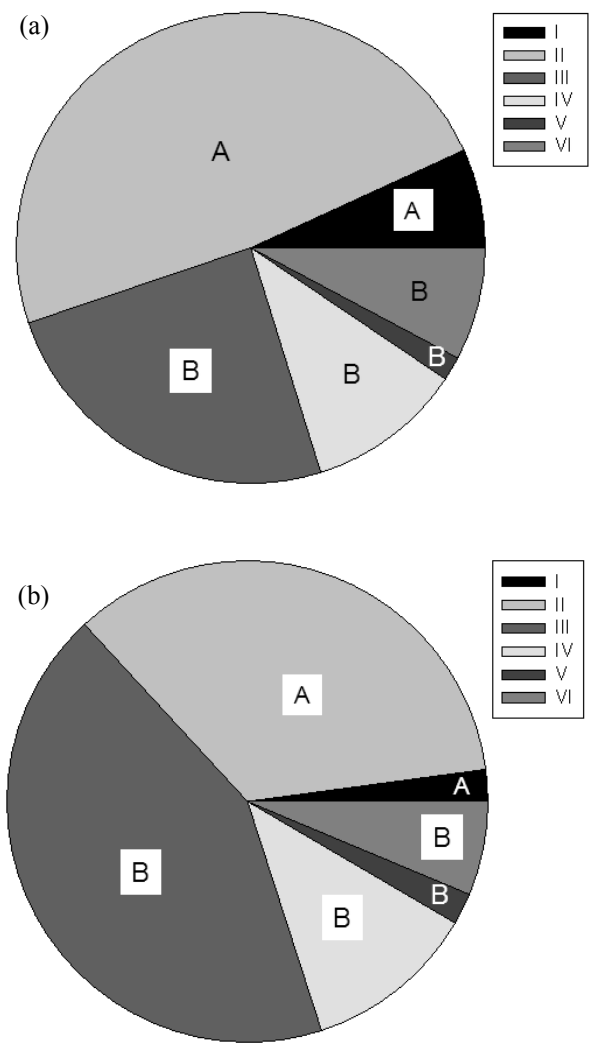

Figure 1: BIRADS grading of the study population (a) and a subgroup of estrogen users (b) Healthy breast (BIRADS I, II) MRI results as 'A' and unhealthy breast MRI results(BIRADS, III, IV, V, VI) as 'B'.

as healthy. Thus, for the subgroup who reported having taken estrogen supplements, $65.33 \%$ of the breast images were classified as unhealthy.

We used SPSS to perform a single-sample binomial test to compare the results of the estrogen users versus the overall study population. The results showed a statistically significant difference $(\mathrm{P}<.001)$, indicating that women taking estrogen or phytoestrogen supplements had a greater tendency to develop breast disease or unhealthy breast conditions.

Based on our experience of assessing over 2000 breast-screening MRI images, we found that an intake of external estrogen is one factor that may enhance proliferative changes in breast glandular tissue. In the current study, cases among the subgroup (experimental group) included women who were using synthetic estrogen for HRT and others who used herbal supplements or Chinese herbs containing estrogen components or phytoestrogen. Our results indicated that natural supplements such as genistein, royal jelly, evening primrose oil, 
Citation: Lin SL, Choy CS, Chan WP, Leung TK (2014) Using Topical Applications of Tamoxifen and a Combination of Phytonutrients Based on Breast MRI to Inhibit Estrogen-Related Proliferation of Human Breast Tissue. Pharm Anal Acta 5: 281. doi: 10.4172/2153-2435.1000281

Page 5 of 10

yam extract, and Ganoderma lucidum could stimulate breast glandular tissue in the same manner as HRT, yielding a positive proliferative change that was detectable in MRI images.

Figures 2 and 3 illustrate selected cases from our observations. As shown in Figure 2, the effects of estrogen or phytoestrogen intake are significant, causing a proliferative change or tumor development. Figure 3 shows obvious improvement after the drug and supplement cessation.

Results of estrogen-enhanced proliferation of MCF-10A, observed under a microscope $(100 \mathrm{X})$ and measured using colorimetric XTT

Estrogen demonstrates the ability to stimulate the proliferation of MCF-10A cells. Morphological changes were observed under 100X power field of a microscope. According to the effects of morphological changes induced by estrogen under a microscope with a $20 \mathrm{X}$ and 100X power field, there was more significant proliferative change by $100 \mu \mathrm{M}$, compared to both $0 \mu \mathrm{M}$ and $10 \mu \mathrm{M}$ of estrogen doses. In addition, according to the colorimetric XTT assay, the percentage of cell proliferation differed significantly when the cells were treated with $100 \mu \mathrm{M}$ of estrogen to $10 \mu \mathrm{M}$ and $0 \mu \mathrm{M}(P<.05)$ (Figure $4 \mathrm{a})$.

\section{Results of tamoxifen/phytonutrient concentration inhibiting cell proliferation of MCF-10A under the effect of estradiol}

Figure $4 \mathrm{~b}$ and $4 \mathrm{c}$ show significant competitive antagonistic effect of tamoxifen $(10 \mu \mathrm{M}$ and $50 \mu \mathrm{M})$ and significant inhibitory effect of the phytonutrients (in different concentrations) on MCF-10a by using estradiol concentrations of $100 \mu \mathrm{mol}$ and $10 \mu \mathrm{mol}$, respectively, and by using a colorimetric XTT assay. The results showed competitive antagonistic effects of tamoxifen $(10 \mu \mathrm{M}$ and $50 \mu \mathrm{M})$ on estrogen

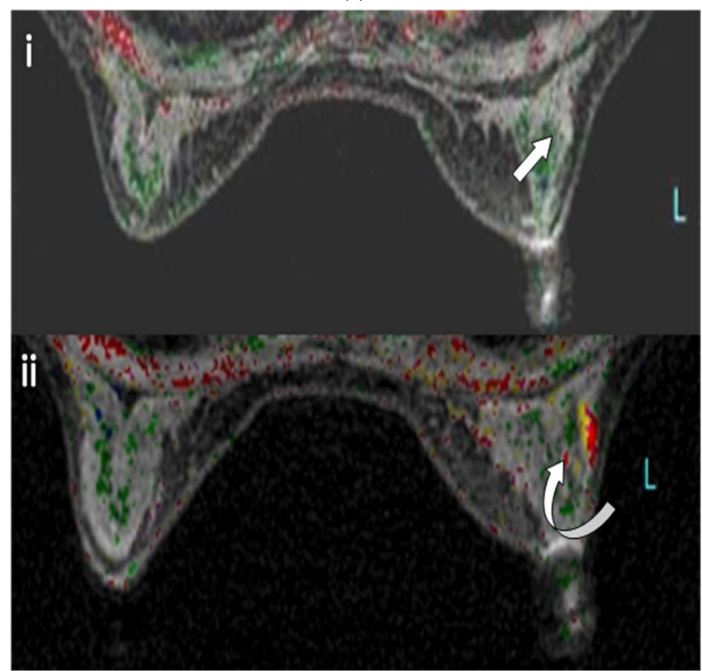

(b)

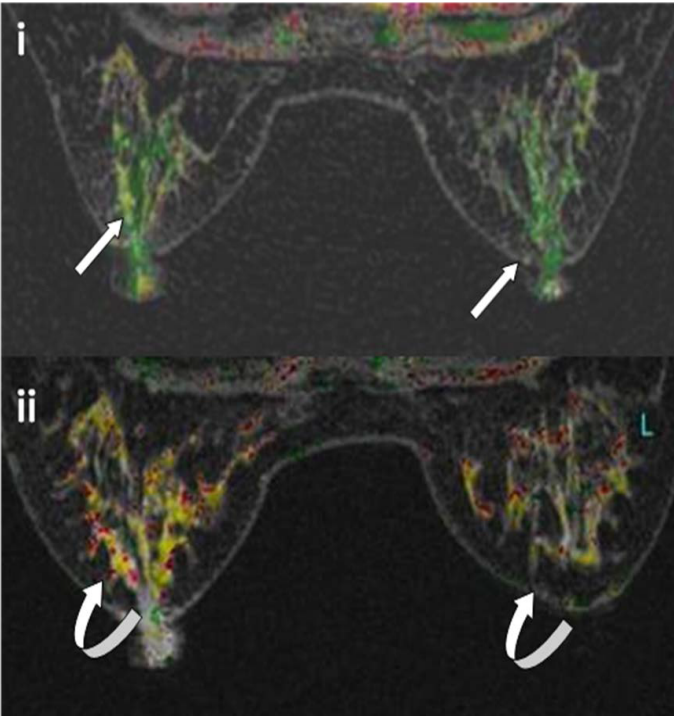

(c)

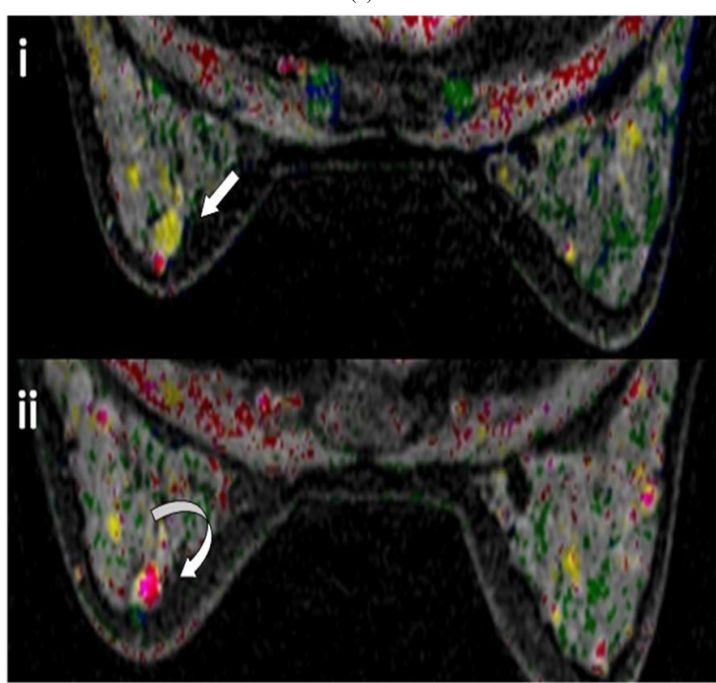

(d)

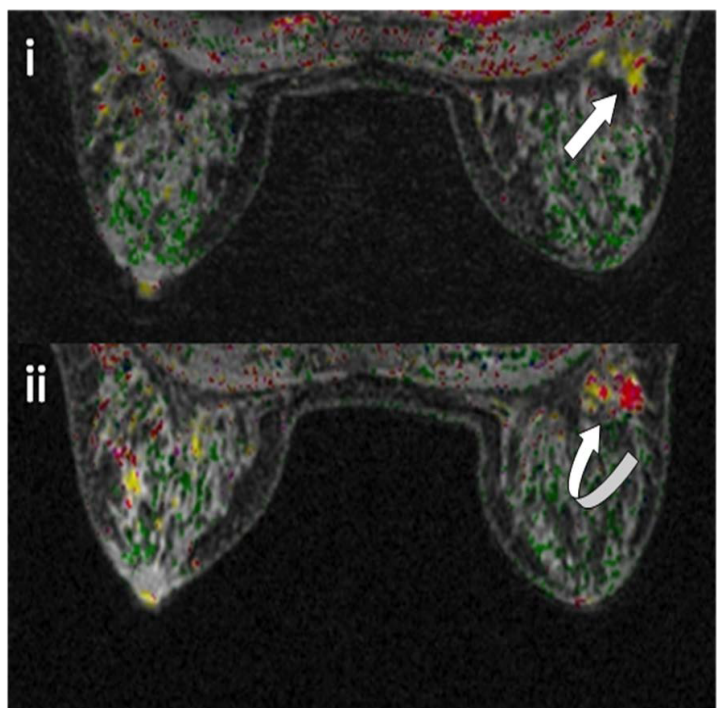

Figure 2: (i) Shows normal signal and color mapping of breast epithelial tissue by performing breast MRI post-processing (straight arrows); (ii) after estrogen therapy or phytoestrogen supplements, a marked abnormal proliferative change of breast epithelial tissue with reddish and yellowish change under color mapping is shown (curve arrows); (a) estrogen therapy; (b) Chinese herb supplement; (c) Chinese herb (manly Chinese Angelica); (d) phytoestrogen supplement, probably a benign small mass (white arrow) with normal yelloish-reddish on color mapping before supplement. 
Citation: Lin SL, Choy CS, Chan WP, Leung TK (2014) Using Topical Applications of Tamoxifen and a Combination of Phytonutrients Based on Breast MRI to Inhibit Estrogen-Related Proliferation of Human Breast Tissue. Pharm Anal Acta 5: 281. doi: 10.4172/2153-2435.1000281

Page 6 of 10

(a)
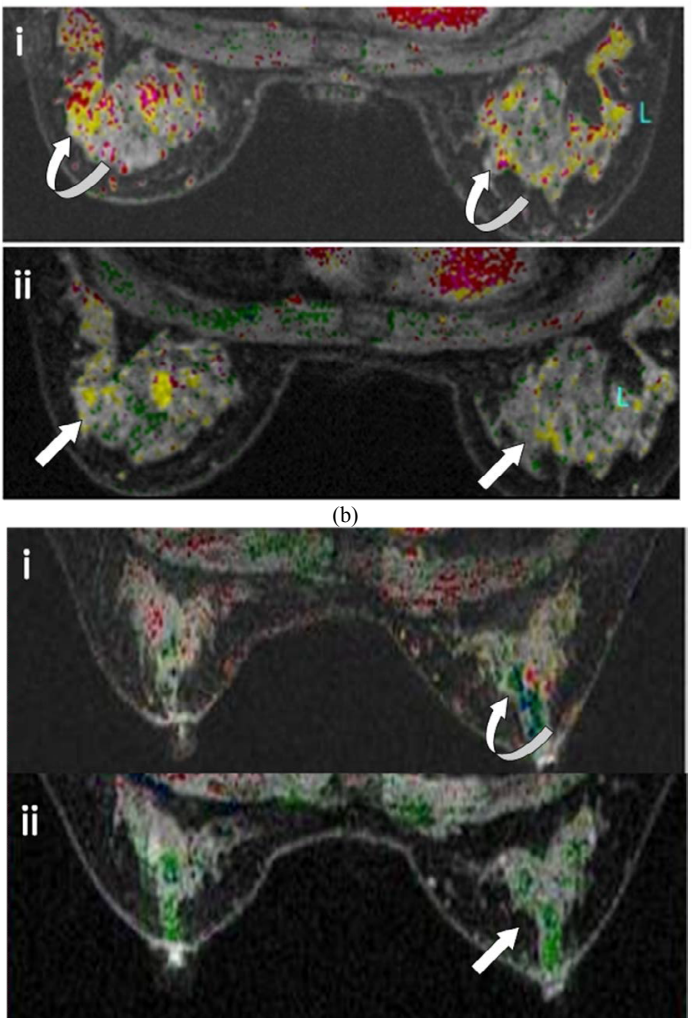

(c)

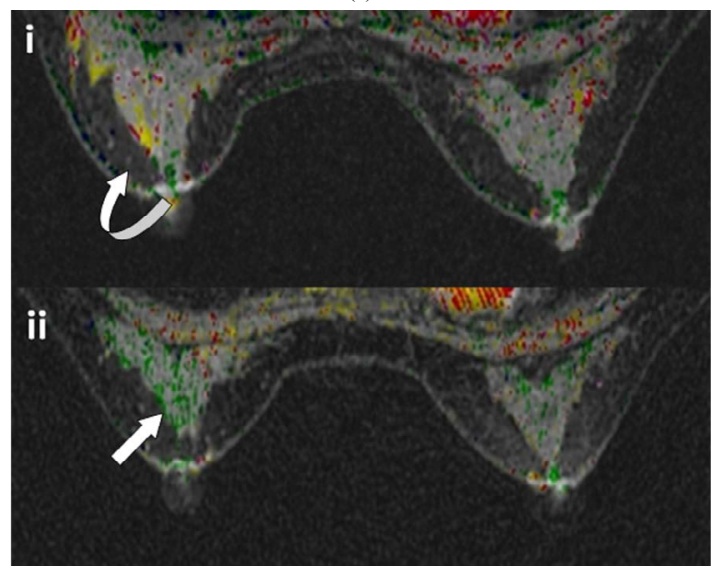

Figure 3: (i) During estrogen therapy or phytoestrogen supplements, diffusely proliferative change of bilateral breast epithelial tissue is represented by reddish and yellowish color parts on color mapping (curve arrows); (ii) after the drug and supplement cessation, MRI pictures show obvious improvement (straight arrows); (a) unknown dietary supplement (including Ganoderma lucidum); (b) royal jelly supplement; (c) hemical estrogen pills, and royal jelly supplement.

$(100 \mu \mathrm{M})$, inducing proliferative stimulation of breast epithelial cells, and positive inhibitory effects of phytonutrients on estrogen $(10 \mu \mathrm{M})$, inducing proliferative stimulation of breast epithelial cells.

Results of transdermal absorption experimental model using franz cell with artificial skin/membrane of tamoxifen/ phytonutrients

This study examined the permeability of tamoxifen in transdermal drug delivery, using the Franz cell experimental model. The results showed that progress increased accumulation of drug through the artificial skin membrane, from the first to the sixth day. These results proved that tamoxifen particles, can pass through artificial skin and membranes without requiring a chemical enhancer (Figure 5a). In addition, a similar result also demonstrated on the phytonutrients (Figure 5b).
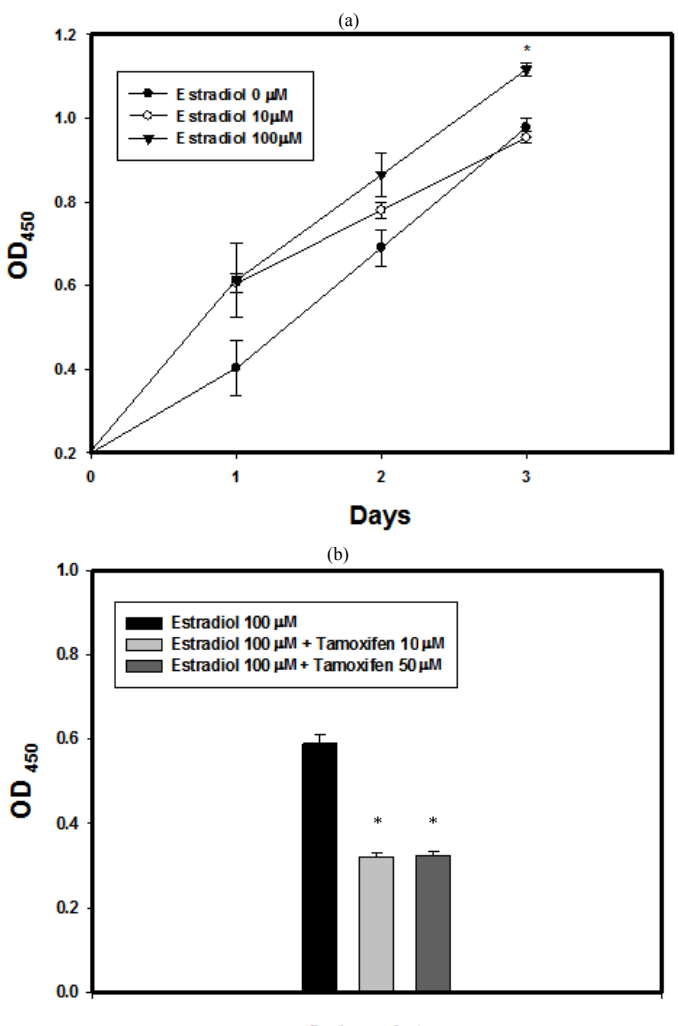

2 days later

(c)
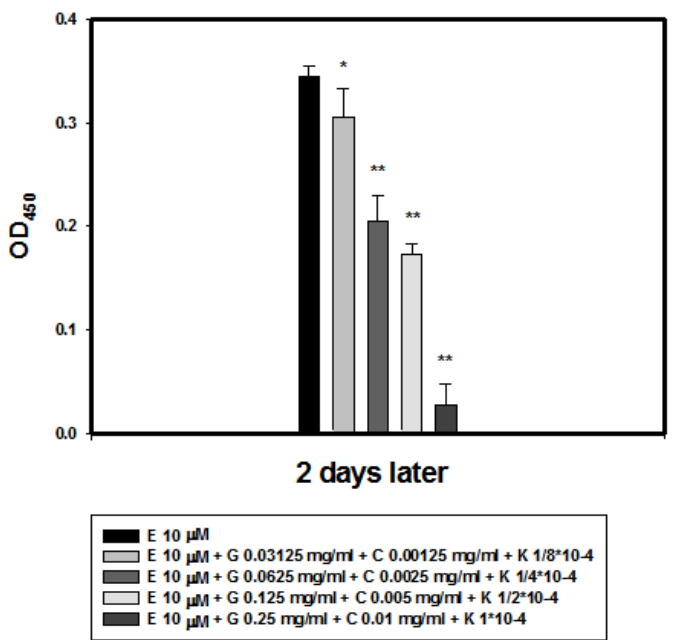

Figure 4: (a) Effects of proliferative change of MCF-10A cells measured by $\mathrm{XTT}$ induced by estrogen. There was more significant proliferative change by $100 \mu \mathrm{M}$, compared to both $0 \mu \mathrm{M}$ and $10 \mu \mathrm{M}$ of estrogen doses. (b) Using $10 \mu \mathrm{M}$ and $50 \mu \mathrm{M}$ of tamoxifen to inhibit proliferative stimulation of estrogen of $100 \mu \mathrm{M}$ on MCF-10a. (c) Using different concentrations of phytonutrients in descending order, to inhibit proliferative stimulation of estrogen of $100 \mu \mathrm{M}$ on MCF-10a. 

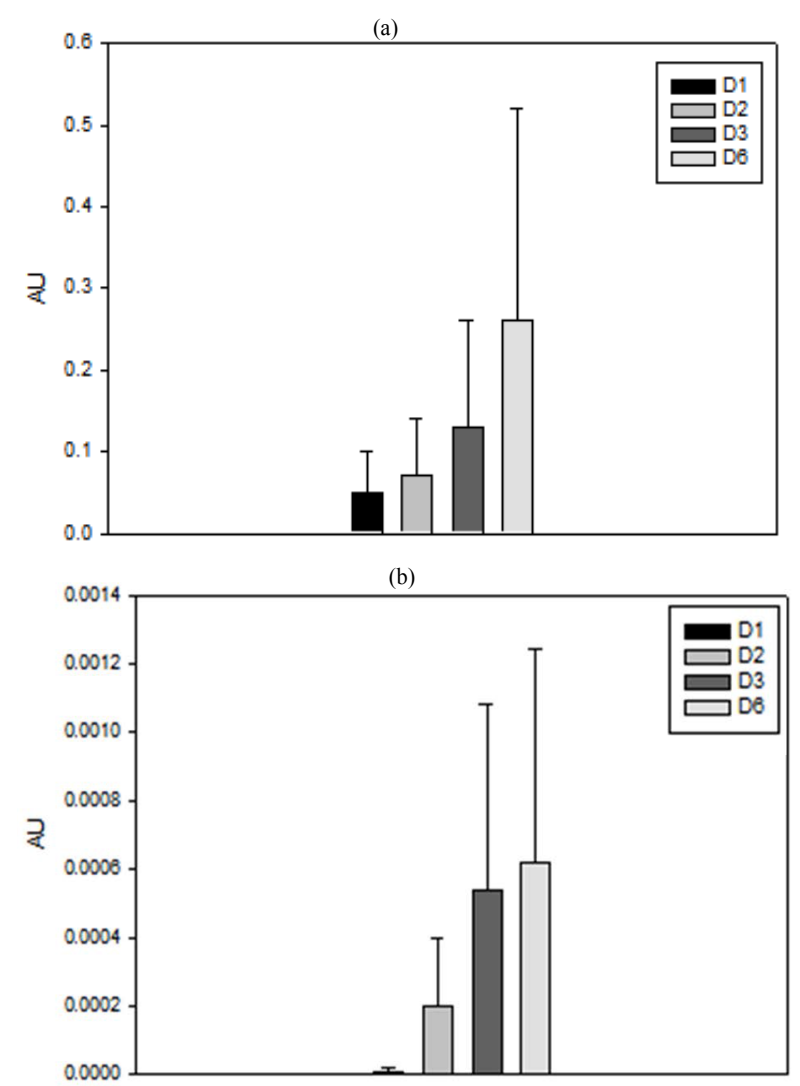

Figure 5: (a) Increased transdermal diffusion of tamoxifen was observed after $1,2,3$ and 6 days $(n=3)$. (b) Increased transdermal diffusion of the combination of phytonutrients was observed after $1,2,3$ and 6 days $(n=3)$.

\section{Result of topical used of tamoxifen/phytonutrients that pass through on skin of rat}

This study examined the ability of tamoxifen and phytonutrients to pass through the full thickness of rat skin by analyzing urine. The results were positive for tamoxifen, but the phytonutrients were undetectable in rat urine (Figure 6).

\section{Results of topical use of tamoxifen/phytonutrient passing through rabbit skin}

This study examined the ability of tamoxifen and phytonutrients to pass through the full thickness of rabbit skin by analyzing venous blood. The results (Figure 7) were positive for both tamoxifen and phytonutrients, which could be detected in rabbit blood.

\section{Result of tamoxifen/phytonutrient retention after topical application on rat's skin}

HPLC showed that positive retentions of tamoxifen (Figure 8a) and phytonutrients (Figure $8 \mathrm{~b}$ ) at the subcutaneous rat skins that maximally on the first day.

\section{Discussion}

Among Taiwanese women, exposure to estrogen has increased in the form of Westernized diets rich in estrogen, an earlier pubertal age than in prior eras, delayed menopause, and obesity after menopause. Estrogen plays a role in promoting glandular cell proliferation, which increases the risk of breast cancer $[18,37,38]$. Some studies have reported

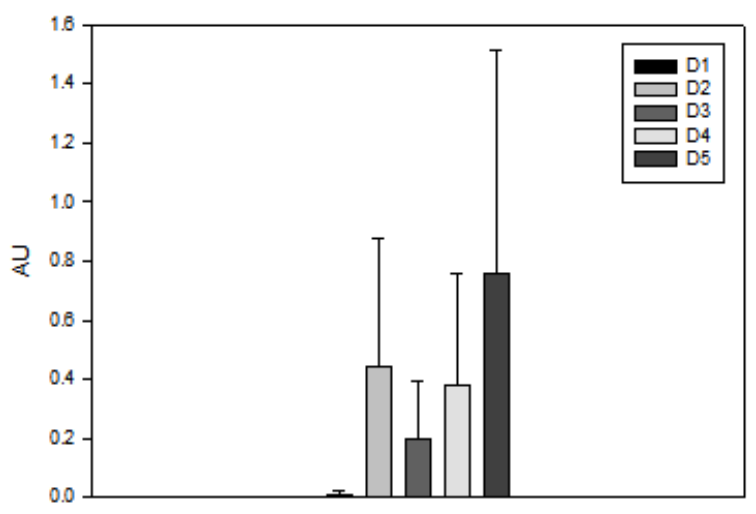

Figure 6: Topical use of tamoxifen applied on the dorsal skin of rats after exposure times of $1,2,3,4$, and 5 days. Samples collected were urine and were analyzed by using HPLC, showing successively increased tamoxifen concentration in the excretion $(n=3)$.

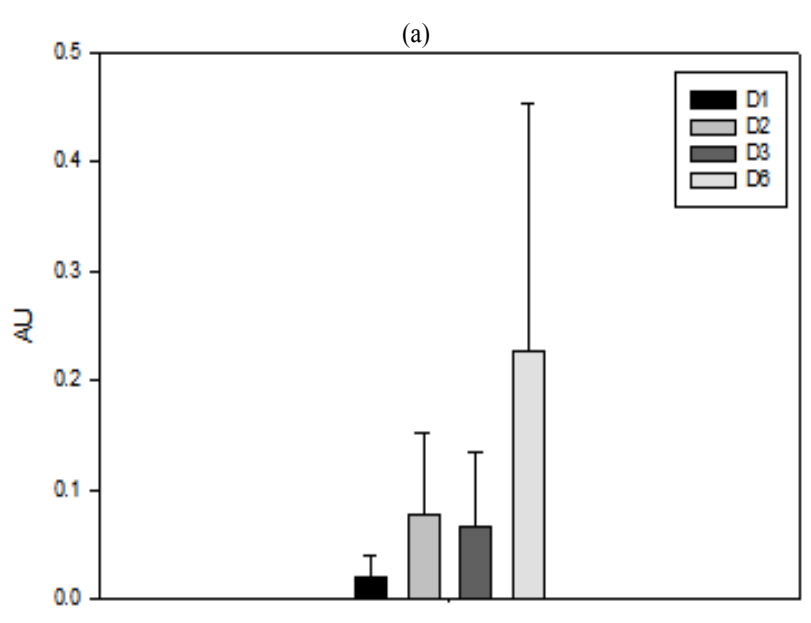

(b)

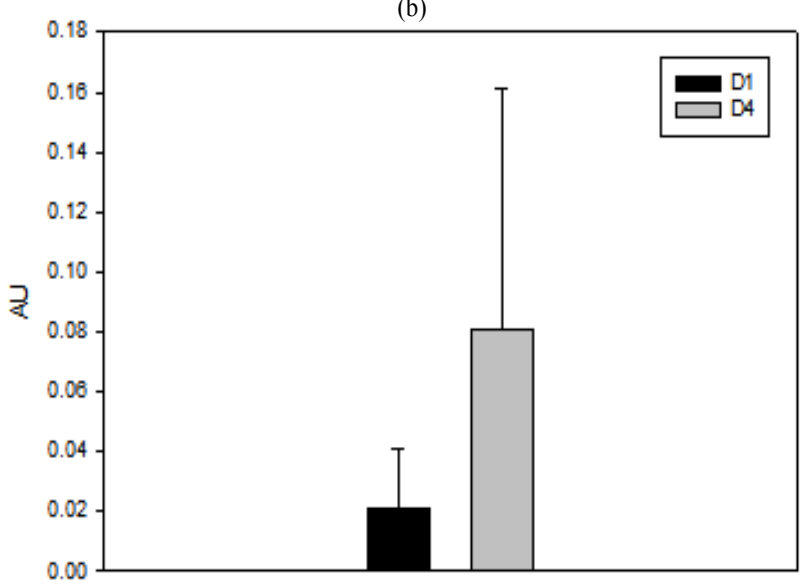

Figure 7: (a) Topical use of tamoxifen applied on the dorsal skin of rabbits after exposure times of 1, 2, 3, and 6 days. Blood samples were collected from the ears, and were analyzed using HPLC, showing successively increased tamoxifen concentration in the blood. (b) Topical use of the combination of phytonutrients applied on the dorsal skin of rabbits that exposure for 1 and 4 days respectively and then collected for analysis were blood from ears, and were send for analysis by HPLC. The results showed the components from the combination of phytonutrients in the blood successively increased. 
(a)

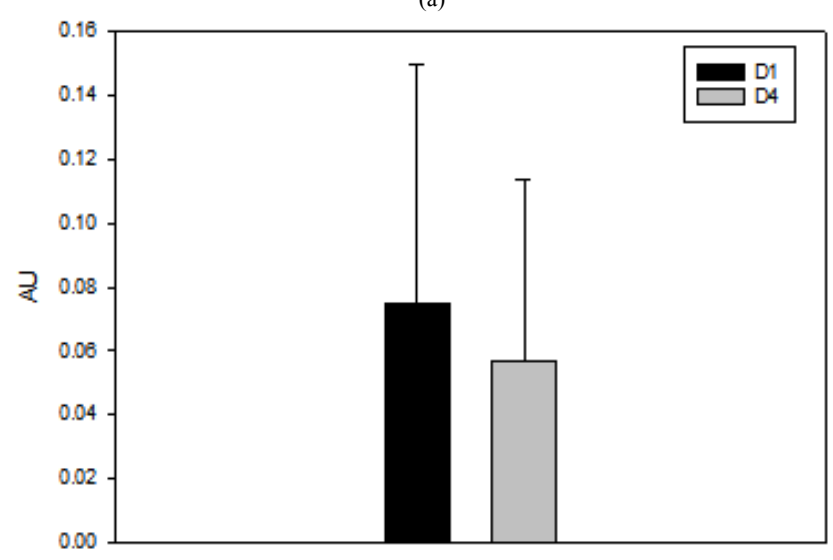

(b)

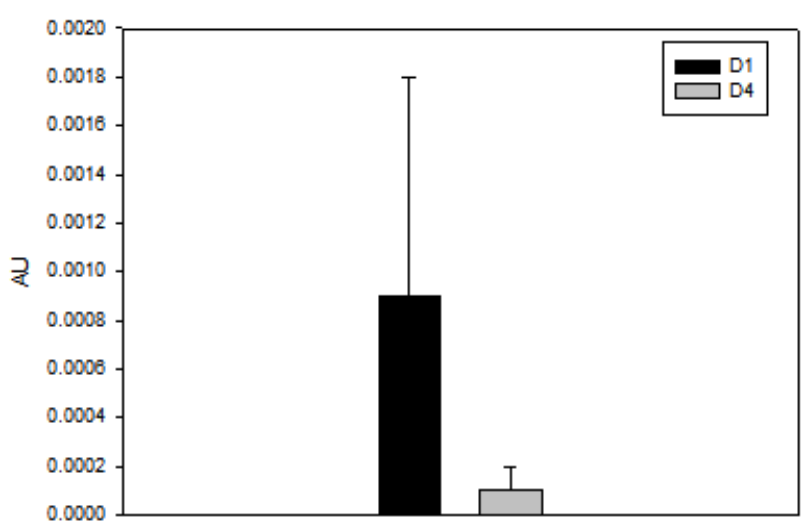

Figure 8: (a) Topical use of tamoxifen applied on the dorsal skin of rabbits after exposure times of 1,2,3, and 6 days. Blood samples were collected from the ears, and were analyzed using HPLC, showing successively increased tamoxifen concentration in the blood. (b) Topical use of the combination of phytonutrients applied on the dorsal skin of rabbits that exposure for 1 and 4 days respectively and then collected for analysis were blood from ears, and were send for analysis by HPLC. The results showed the components from the combination of phytonutrients in the blood successively increased.

that dietary and nutritional factors account for approximately half of all breast cancer cases $[18,39]$. In the past, Asian diets may have facilitated a lower breast cancer rate, but in Taiwan this advantage has disappeared because of the adoption of Westernized diets, over-nutrition, and overuse of herbal or traditional Chinese herbal medicines or other supplements that mimic estrogen components or phytoestrogen $[18,39]$. Women with glandular proliferative disease of the breast are at almost twice the risk of malignancy compared with the general population (including women with non-proliferative lesions). The breast cancer risk for women with atypical hyperplasia (proliferative lesions) is 5 times greater than that for women with non-proliferative lesions. Women with atypical ductal hyperplasia and a family history of breast cancer are at 11 times the risk compared with women who have a non-proliferative lesion and without a positive family history [38]. Breast glandular cells contain cell membrane receptors for estrogen and other receptors for progesterone. Approximately three-fourth of all incidences of breast cancer are estrogen-receptor positive (ER-positive), which means that estrogen causes these tumors to grow. However, during malignant transformation, breast cancer cells do not distinguish between estrogen and phytoestrogen ingested by the patient $[16,17,40]$. Synthetic chemical estrogen (such as xenoestrogen) may take months to metabolize and pass out of the patient's body. The use of chemical estrogen has been found to correlate strongly with an increased incidence of breast cancer. By contrast, most food-grade estrogen supplements or phytoestrogens are metabolized and pass out of the body in several days to weeks. Thus, short-term use of phytoestrogen may be poorly correlated with breast cancer incidence, but long term or overuse of the same products may not be safe. Although extracted from plant tissue, phytoestrogen supplements intended to reduce menopausal symptoms may not provide protection from breast cancer $[12,16,17,40-43]$.

The objective of the first part of this study was to prove the proliferative effect of estrogen and phytoestrogen on breast tissue by performing breast MRI and conducting cell line studies. The second part involved establishing a platform to show the significant proliferative changes of in vitro breast epithelial cell lines under the short-interval effect of estrogen. This platform may be used to show the ability of different anti-estrogen drugs (tamoxifen and phytonutrients), to inhibit the cell proliferation of MCF-10A cell lines. Although MCF 10A cell line is a non-tumorigenic human epithelial cell line with very low ER, we choose MCF 10A as our experimental cell type because it exhibited cell transformation effect by estrogen, through ER- $\beta$-mediated pathway [44]. Tamoxifen have found widespread use in the treatment of breast cancer, as well as preventive agent for breast cancer. It was proved that tamoxifen regulate the activity of other antioxidative enzymes, and afford chemoprotective benefit to ER-containing cells. There were some publications already studied on tamoxifen treatment on MCF-10A without estrogen pretreatment as control group $[45,46]$. Thus, we did not repeat this procedure, only focus on the 'anti-proliferative effect' on estrogen treated MCF-10A after tamoxifen treatment.

Besides, the intention of this study was to develop localized transdermal drug delivery method using minimal dose of tamoxifen and phytonutrients, to prevent breast epithelial cell transformation caused by systemic estrogen effects. Tamoxifen and phytonutrients are generally administered orally. Tamoxifen and phytonutrients undergo extensive hepatic metabolism after oral administration in people. Tamoxifen, despite being relatively effective when administered orally, may exhibits specific side effects such as appetite loss, abdominal cramps, nausea, and vomiting. Therefore, developing a therapeutic system to provide transdermal delivery is beneficial [47]. Previous research on tamoxifen has shown that no metabolic degradation of tamoxifen occurs in the skin; therefore, it is suitable for transdermal delivery [48]. Tamoxifen has an elimination half-life of 5 to 7 days, and its primary metabolite has an elimination half-life of 9 to 14 days. The metabolic and unchanged forms of tamoxifen can be obtained from excretion [47]. Our results are basically meet our initial expectations, tamoxifen and the phytonutrients were successfully pass through skin, slowly decreased concentration in skin and diffused to blood circulation. The result shown in Figure 6 reflected tamoxifen excreted into urine. There was increase in the amount of tamoxifen in rat urine but relative fluctuation with significant error bars are different days. We found tamoxifen available in urine is already met our expectation because the result reflected topical used tamoxifen capable of skin penetration, through blood circulation and finally renal excretion. There are two possible reasons for this imperfect result. Firstly, the tamoxifen in urine from rats' skin may vary by individuals' skin penetration efficiency due to different thickness. Secondly, the treated skin was covered by gauze and protect suit covered on rats' skin, may cause drug absorption different on individual rats. Because this study demonstrated that tamoxifen can be transdermally administered and accumulate subcutaneously over 10 days, it should be evident that topical application provides a more 
Citation: Lin SL, Choy CS, Chan WP, Leung TK (2014) Using Topical Applications of Tamoxifen and a Combination of Phytonutrients Based on Breast MRI to Inhibit Estrogen-Related Proliferation of Human Breast Tissue. Pharm Anal Acta 5: 281. doi: 10.4172/2153-2435.1000281

Page 9 of 10

effective distribution to breast glandular tissue. It is recommended that the minimal dose of tamoxifen application can be employed to inhibit mammary epithelial cell proliferation when possible, thereby reducing its side effects.

In this study, we collected MRI images of the effect of chemical estrogen on women. We also determined that some plant-based foods (such as phytoestrogen) and Chinese herbs influence MRI images similarly. However, this study focused on the estrogenic effect but was limited by unknown mechanisms of plant-based foods (including phytoestrogen) and the Chinese herbs that affect breast epithelial cells. Furthermore, the metabolite mechanisms of each component of the phytonutrients are difficult to monitor. HPLC is limited to detecting and differentiating the components of urea in rat urine after its transdermal absorption. The level of EGCG in urine is usually undetectable in rats; therefore, we expect further investigation of the basic medical research related to this topic [49]. This retrospective study of breast MRI images demonstrated the similarity of cell proliferation induction of breast tissue between synthetic HRT and phytoestrogen. HRT or phytoestrogen supplement intake is crucial for postmenopausal women, localized protection of breasts against the systemic estrogen effect was the primary goal of this research. In this study, we showed that tamoxifen and a combination phytonutrients are effective in suppressing the proliferative effect of estrogen on the human breast epithelial cell line. In addition, tamoxifen and phytonutrients successfully pass through in vitro artificial and in vivo animal skin, and accumulate in subcutaneous skin for at least 5 days. Before clinical application, future work on chemical or surface modifications on the drugs to improve transdermal delivery efficacy is also valuable $[50,51,52]$.

\section{Conclusion}

We believe that the application of tamoxifen and the combination of phytonutrients as a transdermal treatment have significant potential for preventing the estrogen-induced proliferative effect on breast cells that result from estrogen and phytoestrogen supplement use, thereby alleviating future breast cancer development and progression.

\section{Acknowledgment}

This study is funded by Taipei Medical University \& Shuang Ho Hospital (Project Number: 101TMU-SHH-22).The authors are grateful to Dr. Pai-Jung 4 Huang Ms. Jinnia Tsai (AUROA, ASIA), Chuang Chun-Yu, and Chuang Chun-Yu for their dedicated assistance.

\section{References}

1. Rossouw JE, Anderson GL, Prentice RL, LaCroix AZ, Kooperberg C, et al. (2002) Risks and benefits of estrogen plus progestin in healthy postmenopausal women: principal results From the Women's Health Initiative randomized controlled trial. JAMA 288: 321-333.

2. Beral V; Million Women Study Collaborators (2003) Breast cancer and hormonereplacement therapy in the Million Women Study. Lancet 362: 419-427.

3. Tjønneland A, Christensen J, Thomsen BL, Olsen A, Overvad K, et al. (2004) Hormone replacement therapy in relation to breast carcinoma incidence rate ratios: a prospective Danish cohort study. Cancer 100: 2328-2337.

4. Magnusson C, Baron JA, Correia N, Bergström R, Adami HO, et al. (1999) Breast-cancer risk following long-term oestrogen- and oestrogen-progestinreplacement therapy. Int J Cancer 81: 339-344.

5. Stahlberg C, Pedersen AT, Lynge E, Andersen ZJ, Keiding N, et al. (2004) Increased risk of breast cancer following different regimens of hormone replacement therapy frequently used in Europe. Int J Cancer 109: 721-727.

6. Katalinic A, Rawal R (2008) Decline in breast cancer incidence after decrease in utilisation of hormone replacement therapy. Breast Cancer Res Treat 107 427-430.

7. Leung TK, Huang PJ, Chen CS, Lin YH, Wu CH, et al. (2010) Is breast MRI screening more effective than digital mammography in Asian women? J Exp Clin Med 2: 245-250

8. Colditz GA, Hankinson SE, Hunter DJ, Willett WC, Manson JE, et al (1995) The use of estrogens and progestins and the risk of breast cancer in postmenopausal women. N Engl J Med 332: 1589-1593.

9. Pedersen AT, Ottesen B (2003) Issues to debate on the Women's Health Initiative (WHI) study. Epidemiology or randomized clinical trials--time out for hormone replacement therapy studies? Hum Reprod 18: 2241-2244.

10. Beral V, Reeves G, Banks E (2005) Current evidence about the effect of hormone replacement therapy on the incidence of major conditions in postmenopausal women. BJOG 112: 692-695.

11. Roy JA, Sawka CA, Pritchard KI (1996) Hormone replacement therapy in women with breast cancer. Do the risks outweigh the benefits? J Clin Oncol 14: 997-1006.

12. Peeters PH, Keinan-Boker L, van der Schouw YT, Grobbee DE (2003) Phytoestrogens and breast cancer risk. Review of the epidemiological evidence. Breast Cancer Res Treat 77: 171-183.

13. Antoine C, Liebens F, Carly B, Pastijn A, Rozenberg S; Women's Health Initiative (2004) Influence of HRT on prognostic factors for breast cancer: a systematic review after the Women's Health Initiative trial. Hum Reprod 19 741-756.

14. Dietel M, Lewis MA, Shapiro S (2005) Hormone replacement therapy: pathobiological aspects of hormone-sensitive cancers in women relevant to epidemiological studies on HRT: a mini-review. Hum Reprod 20: 2052-2060.

15. Krieger $N(2008)$ Hormone therapy and the rise and perhaps fall of US breast cancer incidence rates: critical reflections. Int J Epidemiol 37: 627-637.

16. Zava DT, Duwe G (1997) Estrogenic and antiproliferative properties of genistein and other flavonoids in human breast cancer cells in vitro. Nutr Cancer 27: 31-40.

17. Hsieh CY, Santell RC, Haslam SZ, Helferich WG (1998) Estrogenic effects of genistein on the growth of estrogen receptor-positive human breast cancer (MCF-7) cells in vitro and in vivo. Cancer Res 58: 3833-3838.

18. Hsieh SC, Lai JN, Lee CF, Hu FC, Tseng WL, et al. (2008) The prescribing of Chinese herbal products in Taiwan: a cross-sectional analysis of the national health insurance reimbursement database. Pharmacoepidemiol Drug Saf 17: 609-619.

19. Barel-Cohen K, Shore LS, Shemesh M, Wenzel A, Mueller J, et al. (2006) Monitoring of natural and synthetic hormones in a polluted river. J Environ Manage 78: 16-23.

20. Åberg UW, Saarinen N, Abrahamsson A, Nurmi T, Engblom S, et al. (2011) Tamoxifen and flaxseed alter angiogenesis regulators in normal human breast tissue in vivo. PLoS One 6: e25720.

21. Sakata M, Ikeda T, Imoto S, Jinno H, Kitagawa $Y$ (2011) Prevention of mammary carcinogenesis in $\mathrm{C} 3 \mathrm{H} / \mathrm{OuJ}$ mice by green tea and tamoxifen. Asian Pac J Cancer Prev 12: 567-571.

22. Love RR (1989) Tamoxifen therapy in primary breast cancer: biology, efficacy, and side effects. J Clin Oncol 7: 803-815.

23. Veprik A, Khanin M, Linnewiel-Hermoni K, Danilenko M, Levy J, et al. (2012) Polyphenols, isothiocyanates, and carotenoid derivatives enhance estrogenic activity in bone cells but inhibit it in breast cancer cells. Am J Physiol Endocrinol Metab 303: E815-824.

24. Yang CS, Chen L, Lee MJ, Balentine D, Kuo MC, et al. (1998) Blood and urine levels of tea catechins after ingestion of different amounts of green tea by human volunteers. Cancer Epidemiol Biomarkers Prev 7: 351-354.

25. Erguvan-Dogan B, Whitman GJ, Kushwaha AC, Phelps MJ, Dempsey PJ (2006) BI-RADS-MRI: a primer. AJR Am J Roentgenol 187: W152-160.

26. Stephens TW (1998) Illustrated Breast Imaging Reporting and Data System (BI-RADS). (3rd edn.) Reston, Va. American College of Radiology.

27. Liberman L, Menell JH (2002) Breast imaging reporting and data system (BIRADS). Radiol Clin North Am 40: 409-430, v.

28. Mario N, Baudin B, Aussel C, Giboudeau J (1997) Capillary isoelectric focusing and high-performance cation-exchange chromatography compared for qualitative and quantitative analysis of hemoglobin variants.Clin Chem. 43 2137-2142. 
Citation: Lin SL, Choy CS, Chan WP, Leung TK (2014) Using Topical Applications of Tamoxifen and a Combination of Phytonutrients Based on Breast MRI to Inhibit Estrogen-Related Proliferation of Human Breast Tissue. Pharm Anal Acta 5: 281. doi: 10.4172/2153-2435.1000281

Page 10 of 10

29. Leung TK, Lee CM, Wu CH, et al. (2012)The protective effect of non-ionized radiation from far infrared ray emitting ceramic material (cFIR) against oxidative stress on human breast epithelial cell (MCF-10A). J Med Biol Eng 2013:In press.

30. Leung TK, Huang PJ, Lee CM, Wu CH, Chen YF, et al.(2013) An experimental model of dynamic mri for tumor enhancement, by using breast cancer cell line heterograft on rat. Biomed Eng Appli Basis Commu 25.

31. Touitou E, Meidan VM, Horwitz E (1998) Methods for quantitative determination of drug localized in the skin. J Control Release 56: 7-21.

32. de Jalón EG, Blanco-Príeto MJ, Ygartua P, Santoyo S (2001) Topical application of acyclovir-loaded microparticles: quantification of the drug in porcine skin layers. J Control Release 75: 191-197.

33. Leung TK, Chu JS, Huang PJ, Lin YH, Lee CM, et al. (2010) Breast MRI for monitoring images of an "adenomyoepithelioma with malignant features", before, during, and after chemotherapy. Breast J 16: 652-653.

34. Leung TK, Huang PJ, Lee CM, Chen CS, Wu CH, et al. (2010) Can breast magnetic resonance imaging demonstrate characteristic findings of preoperative ductal carcinoma in situ in Taiwanese women? Asian J Surg 33:143-149.

35. Leung TK, Huang PJ, Sun LM, Chu JS, Chen CS, et al. (2012) Silicone breast implant with intracapsular rupture coexisting with locally advanced carcinoma. Breast J 18: 76-77.

36. Leung TK, Huang PJ, Liang HH, Hung CS, Chen CS, et al. (2012) Retrospective study of false-positive breast MR images and pathological results in Taiwan. J Exp Clin Med 4: 284-288.

37. Clemons M, Goss $P$ (2001) Estrogen and the risk of breast cancer. N Engl J Med 344: 276-285.

38. Dupont WD, Page DL (1985) Risk factors for breast cancer in women with proliferative breast disease. N Engl J Med 312: 146-151.

39. Tsai AC, Liou JC, Chang MC, Chuang YL (2007) Influence of diet and physical activity on aging-associated body fatness and anthropometric changes in older Taiwanese. Nutr Res27: 245-251.

40. Wang TT, Sathyamoorthy N, Phang JM (1996) Molecular effects of genistein on estrogen receptor mediated pathways. Carcinogenesis 17: 271-275.
41. Keinan-Boker L, van Der Schouw YT, Grobbee DE, Peeters PH (2004) Dietary phytoestrogens and breast cancer risk. Am J Clin Nutr 79: 282-288.

42. Yager JD, Davidson NE (2006) Estrogen carcinogenesis in breast cancer. N Engl J Med 354: 270-282.

43. Duffy C, Perez K, Partridge A (2007) Implications of phytoestrogen intake for breast cancer. CA Cancer J Clin 57: 260-277.

44. Liu S, Lin YC (2004) Transformation of MCF-10A human breast epithelial cells by zeranol and estradiol-17beta. Breast J 10: 514-521.

45. Montano MM, Bianco NR, Deng H, Wittmann BM, Chaplin LC, et al. (2005) Estrogen receptor regulation of quinone reductase in breast cancer: implications for estrogen-induced breast tumor growth and therapeutic uses of tamoxifen. Front Biosci 10: 1440-1461.

46. Montano MM, Chaplin LJ, Deng H, Mesia-Vela S, Gaikwad N, et al. (2007) Protective roles of quinone reductase and tamoxifen against estrogen-induced mammary tumorigenesis. Oncogene 26: 3587-3590.

47. Pathan IB, Setty CM (2011) Enhancement of transdermal delivery of tamoxifen citrate using nanoemulsion vehicle. Int J Pharm Tech Res 3: 287-297.

48. Zhao K, Singh S, Singh J (2001) Effect of menthone on the in vitro percutaneous absorption of tamoxifen and skin reversibility. Int J Pharm 219: 177-181.

49. Amato P, Christophe S, Mellon PL (2002) Estrogenic activity of herbs commonly used as remedies for menopausal symptoms. Menopause 9: 145-150.

50. Kulkarni PV, Roney CA, Antich PP, Bonte FJ, Raghu AV, et al. (2010) Quinolinen-butylcyanoacrylate-based nanoparticles for brain targeting for the diagnosis of Alzheimer's disease. Wiley Interdiscip Rev Nanomed Nanobiotechnol 2: 35-47.

51. Chaturvedi K, Ganguly K, Kulkarni AR, Kulkarni VH, Nadagouda MN, et al (2011) Cyclodextrin-based siRNA delivery nanocarriers: a state-of-the-art review. Expert Opin Drug Deliv 8: 1455-1468.

52. Roney C, Kulkarni P, Arora V, Antich P, Bonte F, et al. (2005) Targeted nanoparticles for drug delivery through the blood-brain barrier for Alzheimer's disease. J Control Release 108: 193-214. 